\title{
Efficacy of Tranexamic Acid in Craniosynostosis Repair in an Infant
}

\author{
Goran Rakić ${ }^{1}$ Danica Stanić ${ }^{1}$ Anna Uram-Benka ${ }^{1}$ Marina Pandurov ${ }^{1} \quad$ Jovana Simin $^{1}$ Biljana Drašković ${ }^{1}$ \\ ${ }^{1}$ Institute for the Healthcare of Children and Youth of Vojvodina, Novi \\ Sad, Serbia \\ Indian J Neurosurg 2015;4:190-192. \\ Address for correspondence Dr. Marina Pandurov, MD, Institute for \\ the Healthcare of Children and Youth of Vojvodina, Hajduk Veljkova \\ 10, Novi Sad 21000, Serbia (e-mail: pandurovmarina@gmail.com).
}

\begin{abstract}
Introduction Extensive blood loss is common in pediatric craniosynostosis reconstruction surgery and so are transfusion-related morbidity and mortality. Tranexamic acid is an antifibrinolytic agent and is increasingly being used in children to reduce perioperative blood loss in various settings, including during craniosynostosis surgery.

Case Report An 8-month-old, 9-kg male infant was admitted to our hospital for craniosynostosis repair. The operation was performed under a balanced general anesthesia. Two central lines and one peripheral line were cannulated in case of a need of massive transfusion. Invasive monitoring was used, as well as prevention of hypothermia. As massive blood loss was expected, before beginning the surgery bolus of tranexamic acid as well as packed red blood cells was administered. During the operation, tranexamic acid was given continuously in an intravenous infusion. The child was hemodynamically stable throughout the operation. After the completion of surgery, which lasted for 5 hours, the patient was extubated in the operating room. Postoperatively, the patient was admitted to the intensive care unit, where he stayed for 24 hours. Hemoglobin values were stable, and there was no need for additional blood replacement.

Keywords

- craniosynostosis

- surgical blood loss

- tranexamic acid

Conclusion Extensive blood loss is common in pediatric craniosynostosis reconstruction surgery, transfusion being unavoidable in the majority of cases. In our patient, tranexamic acid proved effective in reducing perioperative blood loss and transfusion requirement.
\end{abstract}

\section{Introduction}

Craniosynostosis (craniostenosis) is a condition where one or more cranial sutures fuse prematurely leading to focal or global growth delay of the skull. ${ }^{1}$ Surgical treatment involves expansion and remodeling of the cranium in early infancy to prevent increased intracranial pressure, cerebral compression, and blindness. ${ }^{2}$

Because of the risk of massive bleeding, prolonged anesthesia in small children, the possibility of a difficult airway, raised intracranial pressure, venous air embolism, hypothermia, endotracheal tube displacement, positional injuries, corneal abrasions, head and neck edema, and the need of an invasive monitoring, craniosynostosis surgery represents a great challenge for the anaesthetist. ${ }^{2,3}$

Extensive blood loss is common in pediatric craniosynostosis reconstruction surgery and so are transfusion-related morbidity and mortality. ${ }^{2,4}$ Tranexamic acid is an antifibrinolythic agent, increasingly used in children to reduce perioperative blood loss in various settings, including craniosynostosis surgery. ${ }^{2,5-7}$

\section{Case Report}

An 8-month-old, 9-kg male infant was admitted to our hospital for craniosynostosis repair. He had isolated received

August 5, 2015

accepted

September 28, 2015

published online

December 16, 2015
DOI http://dx.doi.org/

10.1055/s-0035-1568999.

ISSN 2277-954X (c) 2015 Neurological Surgeons' Society of India
License terms

$(\circledast) \Theta \circledast$ 
craniosynostosis, without intracranial hypertension or neurological deficiencies. Computed tomographic scan showed absence of large and small fontanelle, as well as a complete parietal synostosis.

The patient was premedicated with $100 \mu \mathrm{g}$ of intramuscular atropine and $200 \mathrm{mg}$ of rectal paracetamol half hour before induction. The induction was inhalational with sevoflurane and oxygen. The patient was intubated with 3.5 sized cuffed endotracheal tube. The Cormack-Lehane view was grade 1 . Right internal jugular vein was cannulated with 22 gauge cannula and right femoral vein with 22 gauge cannula, guided by ultrasound. Invasive arterial blood pressure was monitored via a right dorsalis pedis artery, cannulated with 24 gauge cannula. Peripheral venous access included a 22 gauge cannula in left upper limb. The anesthesia was maintained with oxygen, air, sevoflurane, cisatracurium, and continuous infusion of remifentanyl. The patient was positioned very carefully in the prone position and all intravenous fluids were warmed, as well as the operating room and the patient himself, to prevent hypothermia. There was no possibility to use a precordial Doppler probe to notice venous air embolus, so we had to rely on capnography.

Because massive blood loss was expected, bolus of $400 \mathrm{mg}(50 \mathrm{mg} / \mathrm{kg}$ ) of tranexamic acid was administered before beginning the surgery. During the operation tranexamic acid was given continuously at a rate of 10 $\mathrm{mg} / \mathrm{kg} / \mathrm{h}$. It was difficult to estimate the amount of bleeding; but, since the hemoglobin value was borderline $(110 \mathrm{~g} / \mathrm{L})$ before the surgery, the patient received $15 \mathrm{~mL} / \mathrm{kg}$ of packed red blood cells before surgical incision. The child was hemodynamically stable throughout the operation. After the completion of surgery, which lasted for 5 hours, the patient was extubated in the operating room. Postoperatively, the patient was admitted to the intensive care unit, where he stayed for 24 hours. Hemoglobin values were stable and there was no need for additional blood replacement.

\section{Discussion}

Craniosynostosis surgery is often complicated by massive perioperative bleeding, which can lead to severe hypotension, metabolic acidosis, coagulopathy, acute lung injury, postoperative ventilation, and even cardiac arrest and death. ${ }^{2,8}$ The relative amount of blood volume lost is greater in younger children. This is because the head has a proportionally greater percentage of blood volume and surface area, and therefore, it is prone to more bleeding., ${ }^{49}$ Complications associated with blood transfusion may occur to a greater degree and with a greater frequency in infants than in older children and adults, due to higher oxygen consumption and a higher cardiac output to blood volume ratio. $^{10}$

It is important to know at which moment during the operation, one can expect an extensive blood loss to predict and prepare for hemorrhage. Our patient entered the operating room with borderline values of hemoglobin concentration. As significant hemorrhage occurs during initial scalp dissection and the raising of the periosteum, ${ }^{2}$ we decided for preemptive administration of packed red blood cells $(15 \mathrm{~mL} / \mathrm{kg}$ ) and a loading dose of tranexamic acid (50 mg/kg). Since gradual but significant blood loss can occur throughout the whole procedure and extends into the postoperative period, we decided to give tranexamic acid as an intravenous infusion of $10 \mathrm{mg} / \mathrm{kg} / \mathrm{h}$ during surgery.

It has been proven that tranexamic acid markedly reduces perioperative blood loss and transfusion requirements in children; however, there is significant variability in dosing regimens. Optimal dosage remains uncertain, with wide variations in reported loading doses $(10-100 \mathrm{mg} / \mathrm{kg}$ ) and infusion rates $(1-10 \mathrm{mg} / \mathrm{kg} / \mathrm{h})^{2,7}$

It is difficult to assess the amount of blood volume lost intraoperatively due to child's young age and the fact that a small amount of blood is collected by the suction. Most of the blood lost is absorbed by surgical drapes and the surrounding area. ${ }^{4}$ Because of this, we used hemodynamic parameters, blood gas analysis as well as hemoglobin and hematocrit values as transfusion triggers, which is in accordance with literature findings. ${ }^{4}$ In this case, there was no need for further blood transfusion intra- or postoperatively.

Adverse events have been described in the literature, including seizures as well as thrombotic events. ${ }^{2}$ No adverse events were recorded in our patient.

\section{Conclusion}

Extensive blood loss is common in pediatric craniosynostosis reconstruction surgery, transfusion being unavoidable in the majority of cases. In our patient, tranexamic acid proved effective in reducing perioperative blood loss and transfusion requirement.

\section{References}

1 Thomas K, Hughes C, Johnson D, Das S. Anesthesia for surgery related to craniosynostosis: a review. Part 1. Paediatr Anaesth 2012;22(11):1033-1041

2 Goobie SM, Meier PM, Pereira LM, et al. Efficacy of tranexamic acid in pediatric craniosynostosis surgery: a double-blind, placebo-controlled trial. Anesthesiology 2011;114(4): 862-871

3 Kumar N, Arora S, Bindra A, Goyal K. Anesthetic management of craniosynostosis repair in patient with Apert syndrome. Saudi J Anaesth 2014;8(3):399-401

4 Hughes C, Thomas K, Johnson D, Das S. Anesthesia for surgery related to craniosynostosis: a review. Part 2. Paediatr Anaesth 2013;23(1):22-27

5 Tzortzopoulou A, Cepeda MS, Schumann R, Carr DB. Antifibrinolytic agents for reducing blood loss in scoliosis surgery in children. Cochrane Database Syst Rev 2008;(3): CD006883

6 Schouten ES, van de Pol AC, Schouten AN, Turner NM, Jansen NJ, Bollen CW. The effect of aprotinin, tranexamic acid, and aminocaproic acid on blood loss and use of blood products in major pediatric surgery: a meta-analysis. Pediatr Crit Care Med 2009;10(2):182-190 
192 Efficacy of TXA in Craniosynostosis Repair in an Infant Rakić et al.

7 Kozek-Langenecker SA, Afshari A, Albaladejo P, et al. Management of severe perioperative bleeding: guidelines from the European Society of Anaesthesiology. Eur J Anaesthesiol 2013;30(6):270-382

8 Meyer P, Renier D, Arnaud E, et al. Blood loss during repair of craniosynostosis. Br J Anaesth 1993;71(6):854-857

9 Pietrini D. Intraoperative management of blood loss duringcraniosynostosis surgery, blood conservation strategies in pediatric craniosynostosis surgery. PediatrAnesth. 2013; 23:278-284

10 Barcelona SL, Thompson AA, Coté CJ. Intraoperative pediatric blood transfusion therapy: a review of common issues. Part I: hematologic and physiologic differences from adults; metabolic and infectious risks. Paediatr Anaesth 2005;15(9): $716-726$ 